\title{
Individual Pulse Polarization of Pulsars at Low Frequencies
}

\author{
S.A. Suleymanova, V.A. Izvekova, V.D. Pugachev \\ Astrospace Center of Lebedev Physics Institute, Russia
}

\begin{abstract}
This paper presents the results of the measurements of linear polarization characteristics of individual pulses at frequencies 40,60 and $103 \mathrm{MHz}$ for 10 strong pulsars. We have measured total intensity, linear polarization percentage and angle along the pulse window and obtained distribution displays for these data.

Two orthogonal polarization modes are common features in the radiation of pulsars at low frequency. The energy ratio of these two modes can change significantly between different frequencies. The linear polarisation percentage has a general tendency to increase at low frequencies, but by very different factors for different components of the integrated intensity profile. The relationship between the polarization properties of PSR $0943+10$ and its mode changing behaviour was studied. It is shown that the energy ratio and the frequency of occurence of two polarization modes change simultaneously with changes in the integrated intensity profile.
\end{abstract}

\section{Introduction}

The polarization of radio emission has long been regarded as a phenomenon of critical importance to understanding the pulsar radiation mechanism and magnetic field geometry. The study of individual pulse polarization at the lowest radio frequencies is very important for creating a more complete scenario of pulse emission, particularly from the upper levels of the pulsar magnetosphere.

Several papers have considered polarization behaviour of individual pulses of strong pulsars at decimeter wavelengths. Manchester (1975) describes 12 pulsars mainly at 285, 410 and $1400 \mathrm{MHz}$; Backer and Rankin (1980) present 18 pulsars at $430 \mathrm{MHz}$. Other papers, as a rule, consider some selected pulsars.

We present here a dozen pulsars observed at meter wavelength. Most of them have a counterpart object at decimeters, so one can study the evolution of individual pulse polarization in a significantly wider frequency range.

\section{Observations}

The observation were carried out in 90-s at Pushchino Observatory using radiotelescopes BSA at $103 \mathrm{MHz}$ and DKR-1000 at 60 or/and $40 \mathrm{MHz}$. The measurements of the linear polarization percentage and angle at the arrays of linear polarization are based on measurements of amplitude and phase of overall 
Faraday sinusoidal modulation of the intensity at the output of the multichannel radiometer (Suleymanova et al. 1988). Radiometers with bandwidth $128 \times 20$ $\mathrm{kHz}, 32 \times 5 \mathrm{kHz}$ and $128 \times 1.25 \mathrm{kHz}$ were used to obtain total intensity and polarization parameters for each sample within the pulse window of single pulses as a function of longitude with time resolution 1-7 ms, for diferent frequencies and objects.

\section{Results and discussion}

For 10 pulsars results were obtained for the linear polarization percentage and position angle along the pulse window. These are shown for PSR $0943+10$ in two radiation modes in Figure 1, as an example. Integrated total intensity profile and mean percentage linear polarization of individual pulses are also shown here.

Two orthogonal polarization modes: more intense primary mode (PPM) and secondary (SPM) one - are common features in the radiation of pulsars at low frequency. Similar to data at higher frequencies (Manchester et al. 1975; Backer and Rankin 1980) most pulsars (except 0628-28 and 2217+47) show SPM at one component of the integrated profile or another.

The linear percentage suffers significant variations from pulse to pulse and along the longitude; it is notably higher in those component where SPM is weak or absent: 0031-07(II), 0329+54(IV), 0628-28, 2020+28(I).

The mean percentage linear polarization of individual pulses for different components of the integrated profiles are given in Table I. The observational data at $430 \mathrm{MHz}$ (Manchester et al., 1975; Backer and Rankin, 1980) are given for comparison. The observations of two pulsars $0943+10$ and $2016+28$ at $430 \mathrm{MHz}$ were carried out by authors in the collaboration with J.Rankin and N.Rathnashree in 1992 (Arecibo).

In general, linear polarization of single pulses increases at lower frequencies. On the other hand if one considers integrated pulse components separately then nearly half of them do not show any changes in linear percentage in $40-430$ $\mathrm{MHz}$ frequency range, and the other half show increases of typically 20 percent at low frequencies. Pulsars $0943+10$ and $2020+28$ are clear examples of these two groups.

The evolution of the linear polarization with frequency can be very different for different components of integrated profile. In the case of PSR 0329+54 linear percentage of individual pulses in the range between 103 and $430 \mathrm{MHz}$ remains constant for component I, increases by 20 percent for II and increases by 40 percent for component IV, implying very asymmetrical cone of the polarized radiation at $103 \mathrm{MHz}$.

The relationship between the polarization properties of the radioemission and mode changing phenomenon was studied. The pulsar $0943+10$ is known as a mode-switcher with two distinct shapes: more intense B mode and weaker Q mode (Suleymanova and Izvekova, 1984). Data on PSR 0943+10 at $430 \mathrm{MHz}$ and two low frequencies 40 and $100 \mathrm{MHz}$ are presented on displays in Figure 1. They show that two polarization modes have different distribution over the pulse window for different radiation modes. In B mode the primary, apparently dominant polarization mode spans the entire window, while secondary mode is present only in the main component and in a saddle region. In $\mathrm{Q}$ mode both polarization 

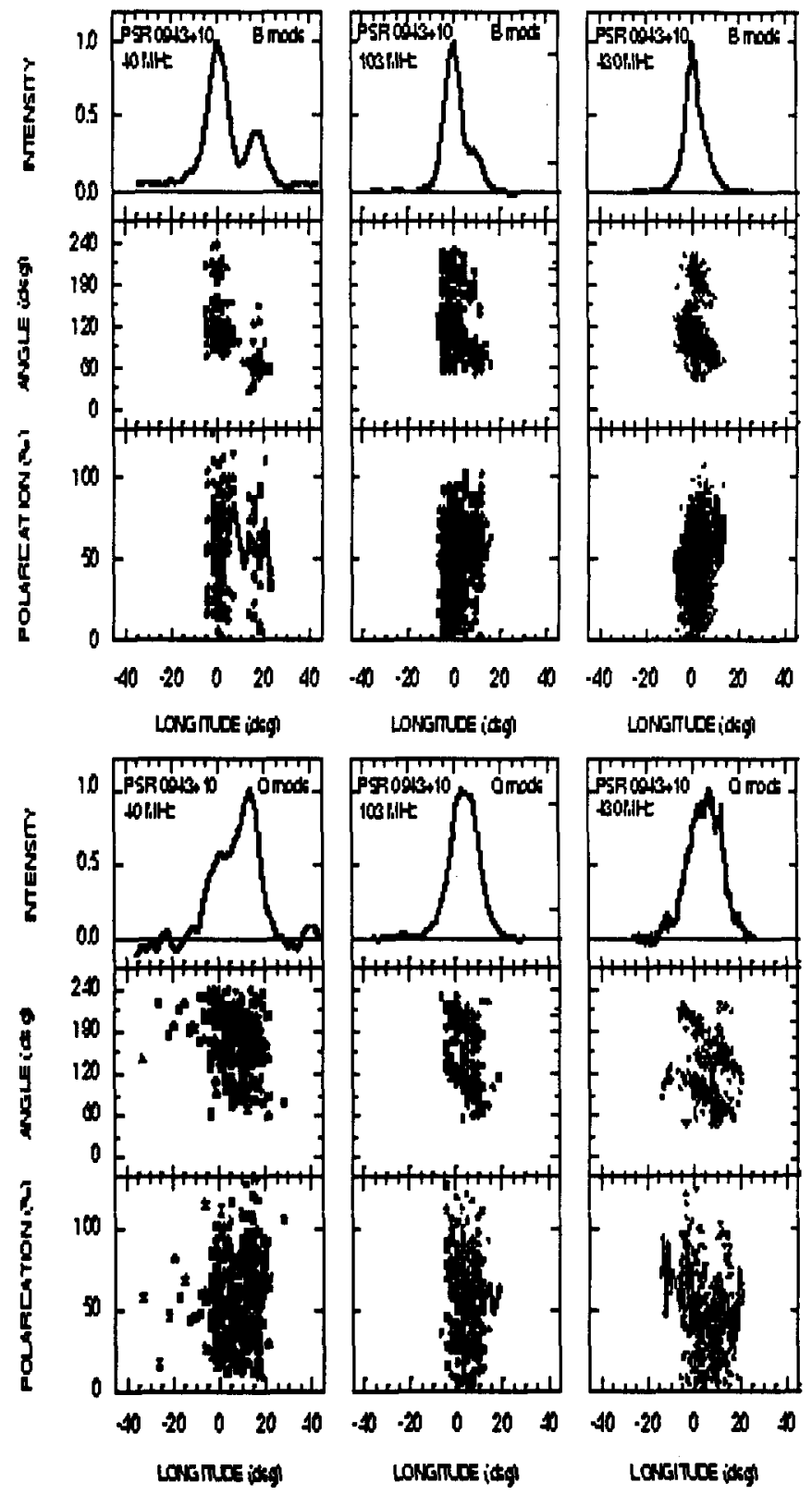

Figure 1. Integrated intensity profile and individual pulse linear polarization angle and percentage distribution displays for PSR $0943+10$. 
modes span the wider range of longitudes, with SPM dominating in radiation of the pulsar. Frequency of occurence ratio is $\mathrm{N}(\mathrm{PPM}): \mathrm{N}(\mathrm{SPM})=0.8: 0.2$ (in $\mathrm{B}$ mode) and 0.4:0.6 (in $Q$ mode). The energy (accumulated intensities) ratio is $\mathrm{I}(\mathrm{SPM}) / \mathrm{I}(\mathrm{PPM})=0.1$ (in B mode) and 1.3 (in Q mode). Thus, our study of $0943+10$ has shown that redistribution of energy of the orthogonal polarization modes along the whole pulse window occurs simultaneously with integrated pulse waveform alterations. The similarity of polarization displays at 40,103 and $430 \mathrm{MHz}$ for every radiation mode on Figure 1 supports the conclusion that switchings between two distinct states of polarization have a broad-band nature.

Table 1. Mean percentage linear polarization of individual pulses

\begin{tabular}{|c|c|cccc|}
\hline \multirow{2}{*}{ PSR } & \multirow{2}{*}{ Components } & \multicolumn{5}{|c|}{ Linear percentage } \\
& & $40 \mathrm{MHz}$ & $60 \mathrm{MHz}$ & $103 \mathrm{MHz}$ & $430 \mathrm{MHz}$ \\
\hline & & $-/-$ & $50 / 50$ & $65 / 85$ & $-/-$ \\
$0031-07$ & I / II & - & - & 55 & - \\
$0320+39$ & & $-/-$ & $-/-$ & $65 / 45 / 90$ & $65 / 25 / 50$ \\
$0329+54$ & I / III / IV & -50 & 75 & 75 & - \\
$0628-28$ & & $40 / 35$ & $30 / 35$ & $-/-$ & $15 / 30$ \\
$0834+06$ & I & $55 / 60$ & $-/-$ & $45 / 50$ & $45 / 50$ \\
$0943+10$ & B / Q & $50 / 50$ & $55 / 50$ & - & $35 / 50$ \\
$1133+16$ & I / II & - & - & 60 & 45 \\
$2016+28$ & I / II & - & - & $95 / 60$ & $75 / 40$ \\
$2020+28$ & & - & - & 50 & - \\
$2217+47$ & & - & \multicolumn{4}{c}{} \\
\hline
\end{tabular}

Acknowledgments. This work was supported by Russian Federal Scientific Program "Astronomy" (Project 3-180) and INTAS (Grant INTAS-94-3097).

S.S. and V.I. approciate very much the financial support of the International Astronomical Union and the Russian Foundation of Basic Researches which have made our participation in IAU Colloquium 160 possible.

\section{References}

Manchester,R.N., Taylor,J.H., Huguenin, G.R. 1975, ApJ, 196, 83

Backer,D.C., and Rankin,J.M. 1980, ApJS, 42, 143

Suleymanova, S.A., Volodin, Yu.V., Shitov, Yu.P. 1988, AZh, 65, 349

Suleymanova, S.A., Izvekova, V.A. 1984, Soviet Ast., 28, 32 